\title{
MODELO DE GERENCIA PARA OPTIMIZAR LA ADMINISTRACIÓN Y GESTIÓN DE RECURSOS DE LOS GOBIERNOS REGIONALES CONCORDANTE CON LA LEY MARCO DE MODERNIZACIÓN DE LA GESTIÓN DEL ESTADO
}

\author{
MODEL OF MANAGEMENT TO OPTIMIZE THE ADMINISTRATION AND \\ MANAGEMENT OF RESOURCES OF THE REGIONAL GOVERNMENTS \\ CONCORDANTE WITH THE LAW FRAMEWORK OF MODERNIZATION OF \\ THE MANAGEMENT OF THE STATE
}

JUAN ANTONIO CHENGUAYEN ROSPIGLIOSI 1

\section{Resumen}

El objetivo fue formular un modelo de gerencia para optimizar la gestión y administración de recursos en los gobiernos regionales. El diseño fue no experimental. La muestra de estudio estuvo constituida por 30 funcionarios de los gobiernos regionales. Se utilizó un cuestionario y una ficha de recolección de datos, construidos y validados para los fines del estudio. Entre los principales resultados se encontró que la percepción del rol gerencial desplegado en los gobiernos regionales está desfasado y no contribuye a la gobernanza $(53,3 \%)$ y que es prioritaria como parte del diseño organizacional $(46,7 \%)$; y las principales razones que no permitirían una adecuada ejecución de los recursos es a que prevalecen intereses políticos $(33,3 \%)$ y personal desmotivado $(26,7 \%)$. Conclusión: Se formula un modelo de gerencia para optimizar la gestión y administración de recursos en los gobiernos regionales, con un nuevo enfoque organizacional

Palabras clave: Modelo de gerencia / gestión /administración/Gobierno Regional

\section{Abstract}

The objective was to formulate a management model to optimize the management and administration of resources in regional governments. The design was not experimental. The study sample was constituted by 30 officials of the regional governments. A questionnaire and a data collection form were used, constructed and validated for the purposes of the study. Among the main results, it was found that the perception of the managerial role deployed in regional governments is outdated and does not contribute to governance $(53.3 \%)$ and that it is a priority as part of the organizational design (46.7\%); and the main reasons that would not allow an adequate execution of the resources is to prevail political interests $(33.3 \%)$ and unmotivated personnel $(26.7 \%)$. Conclusion: A management model is formulated to optimize the management and administration of resources in regional governments, with a new organizational approach

Keywords: Management / management / administration model / Regional Government

\section{INTRODUCCIÓN}

En julio del 2002 se promulga de Ley № 27783, Ley de Bases de la Descentralización, que tiene objeto desarrollar "el Capítulo de la Constitución Política sobre Descentralización, que regula la estructura y organización del Estado en forma democrática, descentralizada y desconcentrada, correspondiente al Gobierno Nacional, Gobiernos Regionales y Gobiernos Locales". Más adelante, el 7 de marzo de 2002, se promulga la Ley $\mathrm{N}^{\circ} 27680$, Ley de Reforma Constitucional del Capítulo XIV del Título IV sobre Descentralización, en la que en su artículo 188

1 MAGISTER EN ADMINISTRACIÓN Y DIRECCIÓN DE EMPRESAS 
precisa que la descentralización es una forma democrática y constituye una política permanente de Estado. Posteriormente, se presenta en el 2011 el Plan Nacional de Descentralización 2012-2016, con el propósito de delinear una ruta clara para continuar el proceso de descentralización y "procurar el desarrollo integral, equilibrado y sostenible de nuestro país" (Presidencia del Consejo de Ministros, 2011,5). Al realizar un balance del proceso de descentralización, se evidencia un avance importante, sin embargo, no se aprecia resultados óptimos (Ángeles, 2012,17). La situación delineada, motivó la presente investigación, a fin de proponer un Modelo de Gerencia que permita optimizar la administración y gestión de los recursos en el ámbito de los gobiernos regionales, en un contexto normativo, indudablemente propicio, como lo es la Ley Marco de Modernización de la Gestión del Estado Peruano.

Es necesario, subrayar que el Perú, en los últimos diez años, logró una de las tasas más altas de crecimiento económico de la región (Banco Mundial, 2014), sin embargo, "este crecimiento económico, no fue acompañado por un crecimiento similar de la capacidad del Estado ...." (Perú - Presidencia del Consejo de Ministros $(2013,9)$. En este sentido, se identificaron deficiencias de la gestión pública, las que según Levitsky (2012) sintetiza al afirmar que "....el Perú tiene uno de los estados más disfuncionales de América Latina. Los gobiernos locales y regionales carecen de un mínimo de capacidad administrativa. $Y$ la seguridad y la justicia - funciones básicas del Estado - no son ni mínimamente garantizadas". Actualmente, se cuestiona constantemente la gestión de los gobiernos regionales, enfocada en "una asignación basada en insumos, el presupuesto centrado en la oferta institucional, distanciamiento de la planificación y de los objetivos estratégicos, alejada de los problemas centrales de la ciudadanía y de la demanda" (USAID, 2011) lo que denota una gestión poco flexible, desarticulada, centralista y carente de transparencia (Llona, 2011).

A partir de enero del 2002, el Estado peruano buscó establecer los principios y la base legal para iniciar un proceso de modernización, la misma que fue materializada con la Ley $\mathrm{N}^{\circ}$ 27658, Ley Marco de Modernización de la Gestión del Estado. Sin embargo, después de más de doce años, esta transferencia de funciones y la disponibilidad de recursos, no han sido acompañadas de "las capacidades de gobierno y de gerencia suficientes para proveer más y mejores bienes y servicios públicos a todos los ciudadanos en todo el país, según su necesidad " (Perú - Presidencia del Consejo de Ministros, 2013,11). En el Perú, Morales O., Barrera A., Rodríguez M., Romero C. y Távara R. (2014), identificaron como elementos esenciales del proceso de gestión de la innovación: la motivación, los actores, los recursos, las alianzas, la ciudadanía y el cambio cultural. Jiménez H. (2010) afirma que la regulación y/o control de la gestión de intereses surge como una medida que ayuda a transparentar la actuación de la administración pública frente a la gestión de los intereses particulares. En Venezuela, Peraza J. (2010) investigó sobre una propuesta de un modelo gerencial estratégico socialmente responsable. Encontró que algunos municipios presentaron: falta de recursos financieros, presupuestarios, desarrollo y capacitación del capital humano, intromisión distorsionadora de la política partidista, poca responsabilidad social comprometida con los ciudadanos y aplicación de estrategias. En la misma nación, Romero M. (2010) realizó el estudio Reforma de Estado, Gerencia Pública Social y Organizaciones No Lucrativas. Reflexiones en torno a Venezuela. Concluye respecto a la gerencia pública social, que el orden constitucional de 1999, promovió la participación en los asuntos públicos más allá de los procesos electorales, así como una intervención directa de las organizaciones sociales en la gestión y Guzmán J. y Tortolero F. (2013) en su investigación concluyeron que las nuevas técnicas de la comunicación influyen en el manejo de las organizaciones ya que contribuyen de forma positiva al mejoramiento, aplicación y entendimiento de las mismas.

Según Oyarce (2012) la noción de un trabajo administrativo tradicional limitado al cumplimiento de lo legalmente asignado, era insuficiente frente a los desafíos de la gerencia pública, por lo que se suscitaron diversas reformas en la década de los 80 y 90 , lo que devino en una nueva concepción denominada la Nueva Gerencia Pública, la cual busca mejorar la capacidad de administrar de los gestores públicos y mejorar la productividad y la eficiencia pública. 


\section{OBJETIVOS}

a) Identificar cuál es la percepción del rol gerencial, qué razones no permitieron una adecuada ejecución y precisar cuál es la percepción de necesaria vinculación entre la Secretaría de Descentralización del Presidencia del Consejo de Ministros y la Asamblea Nacional de Gobiernos Regionales desplegado en los gobiernos regionales. b) Precisar cómo fue la inversión pública en el Perú, identificar cuál fue el porcentaje de ejecución presupuestal, la capacidad de ejecución global, comportamiento de los indicadores de salud acceso a telecomunicaciones, educación, empleo, y competitividad 2013-2014 que presentaron los gobiernos regionales en el Perú.

\section{METODOLOGÍA}

Investigación no experimental, descriptiva. El Ámbito de estudio fueron los Gobiernos Regionales de San Martín, Cuzco, Huánuco, Lambayeque, Arequipa. La población diana estuvo conformada por autoridades de los Gobiernos Regionales y funcionarios. Para realizar la medición de la variable se utilizó una ficha de cotejo y un cuestionario de 14 ítems, previo proceso de validación mediante prueba de expertos (Método Delphi). Se coordinó con los Gobernadores Regionales y Gerentes de los Gobiernos Regionales que constituyen la muestra. Para el tratamiento de los datos se utilizó estadística descriptiva.

\section{RESULTADOS}

Más de la mitad de los funcionarios perciben que el rol de la gerencia dentro del marco de la normativa vigente, se encuentra desfasado y no contribuye con la gobernanza; sin embargo, un $26,7 \%$ considera que permite una mejor gestión y control de los recursos públicos (Fig. 01). Cabe resaltar, que si bien es cierto que más de la mitad de los funcionarios evidencian que el rol de gerencia que se despliega no es coherente en general, continúan el ritmo y cadencia laboral que impone la situación, ya que entienden que su cambio está ligado a la voluntad política e incluso legislativa, lo que muchas veces deviene en un estado de desmotivación laboral.

Fig. 01: Percepción de Gestión

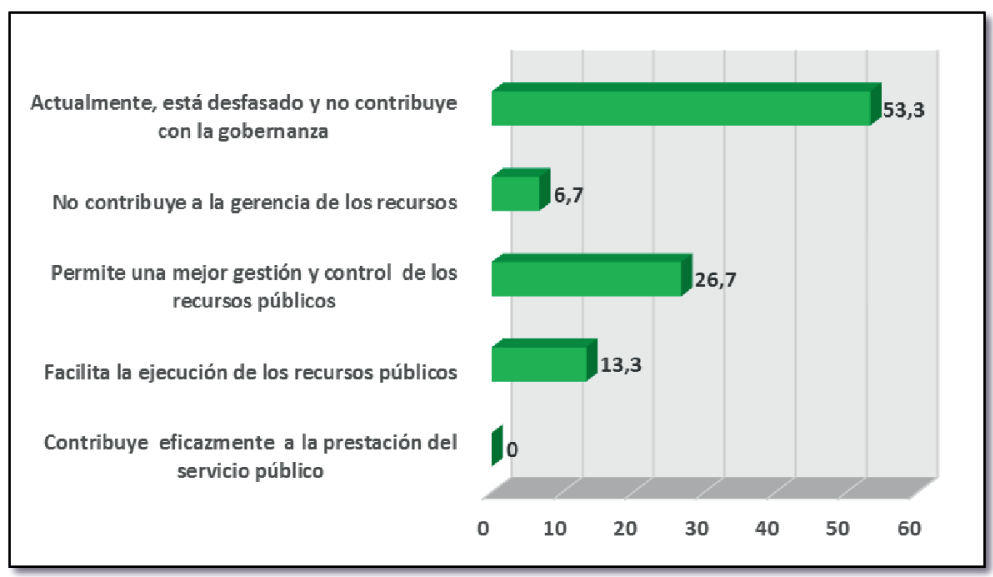

Respecto a la relevancia que se le atribuye a la presencia gerencial en la secuencia del diseño organizacional estructurado con sujeción a la naturaleza, objetivos, misión, visión, fines y funciones de los gobiernos regionales, los funcionarios públicos en un $46,7 \%$ consideran que es prioritaria, el $13,3 \%$ considera que no. Las razones que no permitirían una adecuada ejecución de los recursos, la tercera parte $(33,3 \%)$ consideraron que prevalece intereses políticos; un $26,7 \%$ opina que se debe mayormente a que el personal está desmotivado, lo que no coadyuvaría al cumplimiento cabal de sus funciones (Fig 02) 
Fig. 02: Razones que no permitirían una adecuada ejecución de recursos

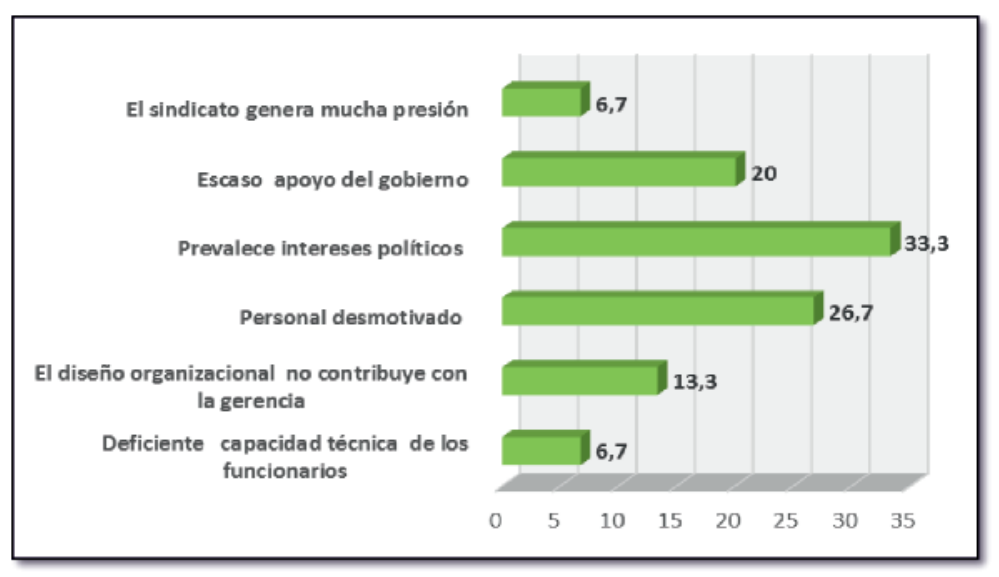

Los Gobiernos Regionales tienen competencias de carácter constitucional, como también competencias exclusivas y compartidas establecidas en la Constitución Política del Estado, la Ley Orgánica de Gobiernos Regionales (Ley N ${ }^{\circ} 27867$ ) y la Ley de Bases de la Descentralización (Ley $N^{\circ} 27783$ ), sin dejar de lado, las competencias delegadas acordadas entre el Gobierno Nacional y el Gobierno Regional respectivo ( Artículo $13^{\circ}$ de la Ley $\mathrm{N}^{\circ}$ 27783). En este contexto, la percepción de los funcionarios relacionada con la necesaria vinculación entre la Secretaría de Descentralización y la Asamblea Nacional de Gobiernos Regionales para el cumplimiento del rol gerencial, es de muy necesaria la vinculación en casi las dos terceras partes $(63,3 \%)$. Sin embargo, la diferencia $(36,7 \%)$ la considera innecesaria. Las razones por las que no se

Fig. 03. Inversión pública (Millones de nuevos soles)

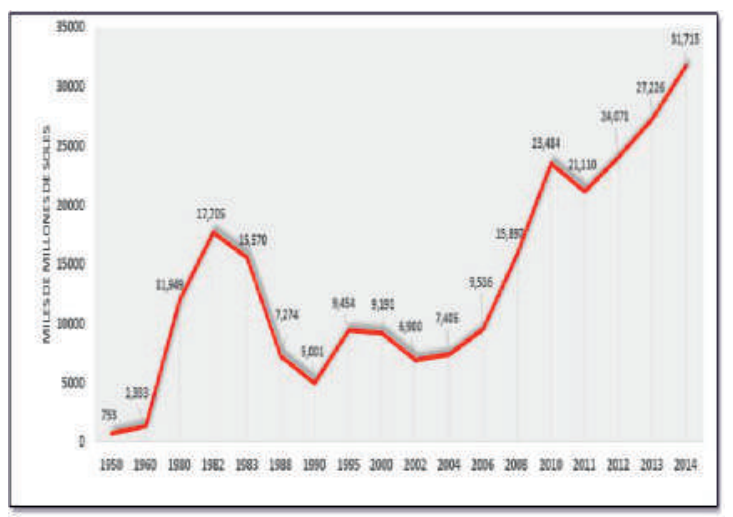

El Perú está ubicado entre las economías con mayor crecimiento en el mundo después de la China e India y menor inflación (Castilla, 2012). Las principales transferencias corrientes a los Gobiernos Regionales, son por concepto de concretó una vinculación efectiva, el 63,2\% consideró que la razón fundamental fue que depende de la decisión política del gobierno; mientras que un $36,8 \%$ piensa que frecuentemente no hay toma de decisiones compartidas entre ambas.

Respecto a la inversión y ejecución de la inversión pública durante el periodo de 1950 al 2014, esta se incrementó considerablemente, especialmente a partir del 2008, con 15,897 millones de nuevos soles, es decir se incrementó 6381 millones de nuevos soles respecto al año 2006. En el 2014, la inversión en millones de nuevos soles alcanzó la cifra de 31,715 , lo que se corresponde con un $6,4 \%$ de inversión pública con respecto al $\mathrm{PBI}$, lo que resulta positivo para el Perú (Fig. 03 y 04).

Fig. 04. Inversión pública en relación al PBI

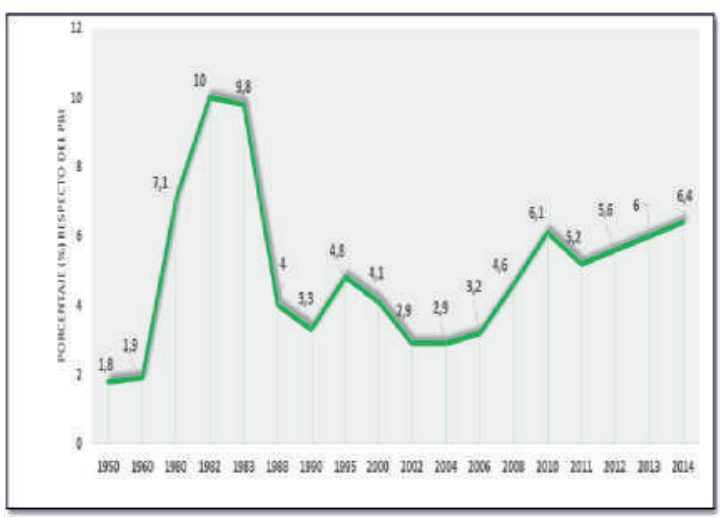

Canon minero, FONCOMUN y regalías mineras, las que presentan una tendencia a incrementarse en el periodo 2007-2011. Empero, a pesar de que el Estado ha transferido cada trimestre y cada año, mayores recursos a los 
Gobiernos Regionales, el impacto en términos de indicadores sociales, educativos, de salud, empleo, etc., debido a varios factores, pero el factor a que nuestro juicio resulta relevante es que no se ha desplegado una adecuada gerencia que conduzca el norte del gasto y defina los criterios mínimos del mismo, lo que consecuentemente ha encarecido el manejo de los recursos públicos.

En las 491 unidades ejecutoras de los Gobiernos Regionales, no se ejecutan todos los recursos que se transfieren, lo que en la praxis significa que se deja de atender necesidades de la población de cada región y se revierte al estado los recursos no ejecutados. Los resultados de la ejecución de los recursos, evidencian que no se tiene presencia de la gerencia, y se piensa que resaltando la labor en la prestación del servicio público lo demás vendrá por defecto, sin embargo, en la actualidad no se otorga un buen servicio público ni se ejecutan los recursos para hacerlo.

La ejecución de la inversión pública se incrementó en el periodo 2011-2016 comparativamente respecto de los periodos anteriores (2001-2006 y 2006-2011. Presumiblemente, el crecimiento de la ejecución de la inversión pública, se debería al incremento en el presupuesto designado para los proyectos de inversión pública y una mejor capacidad de ejecución (Fig. 05)

Fig. 05: Capacidad de Ejecución Global de la inversión pública

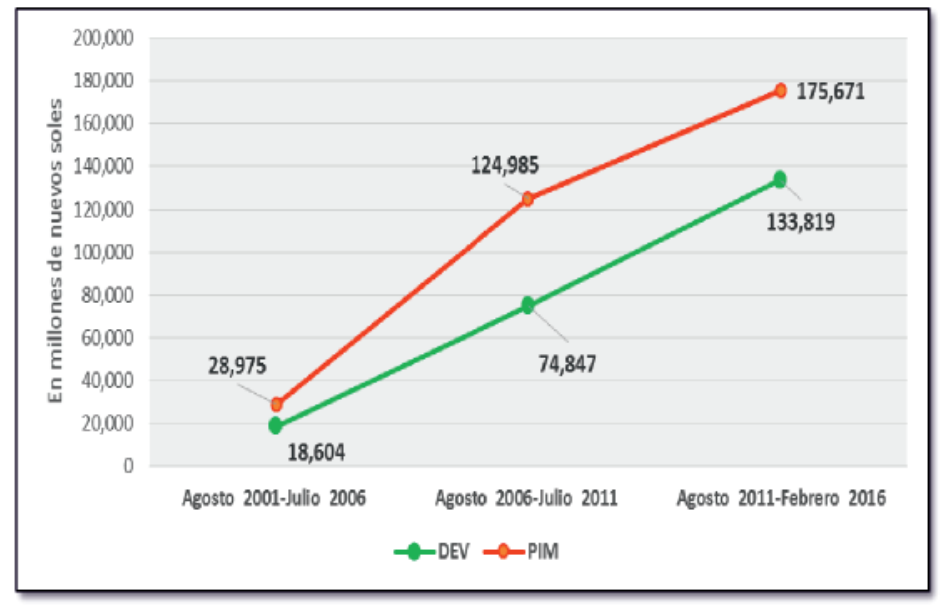

Se aprecia que la ejecución de la inversión pública en el 2014 (Tabla 11) ha alcanzado porcentajes de $79 \%$ para el Perú, 88\% para el gobierno nacional; $77 \%$ para los gobiernos regionales y $72 \%$ para los gobiernos locales de avance de ejecución del presupuesto programado, lo que constituye un logro histórico de ejecución comparado con los registros finales de los años 2007 al 2013 , a excepción de los gobiernos regionales, ya que en el 2013 alcanzaron un punto porcentual más $(78 \%)$ que el año 2014 (77\%).

Tabla 11: Eficiencia en la ejecución de la inversión pública

\begin{tabular}{|c|c|c|c|c|}
\hline Año & Perú (\%) & Gobierno Nacional (\%) & $\begin{array}{l}\text { Gobiernos } \\
\text { Regionales } \\
\text { (\%) }\end{array}$ & $\begin{array}{l}\text { Gobiernos } \\
\text { Locales } \\
\text { (\%) }\end{array}$ \\
\hline 2007 & 52 & 64 & 50 & 42 \\
\hline 2008 & 53 & 54 & 48 & 55 \\
\hline 2009 & 63 & 80 & 54 & 58 \\
\hline 2010 & 72 & 81 & 61 & 73 \\
\hline 2011 & 67 & 81 & 61 & 59 \\
\hline 2012 & 71 & 82 & 76 & 63 \\
\hline 2013 & 73 & 81 & 78 & 66 \\
\hline 2014 & 79 & 88 & 77 & 72 \\
\hline
\end{tabular}


A la par del auge económico en el Perú en los últimos años, también se observa en general, una mejora de los indicadores de pobreza y condiciones de vida. Se aprecia una detracción mayor del porcentaje de la población en situación de pobreza urbana (Año 2004: 4,2\%; Año 2011: $18 \%$ ) en comparación que la pobreza en zona rural (Año 2004: 83,4\%; Año 2011: 56,1\%). Respecto al porcentaje de la población en situación de pobreza extrema igualmente, los indicadores muestran una disminución importante en la zona urbana (Año 2004: 5,7\%; Año 2011: 1,4\%), mientras que, en la zona rural, también disminuyó, pero en menor proporción respecto a la urbana (Año 2004: 41,6\%; Año 2011: $20,5 \%$ ). En cuanto a las condiciones de vida, como ingreso real promedio per cápita se elevó de 512,5 en el año 2004 a 721,2 en el 2011. Respecto al acceso a los servicios básicos, el porcentaje de hogares sin acceso a alumbrado público, disminuyó considerablemente más (14 $\%$ que las otras categorías de acceso a servicios.

El acceso a las telecomunicaciones, experimentó un incremento significativo, especialmente el porcentaje de hogares con al menos un miembro que accede al teléfono celular, ya que $16,4 \%$ en el 2014 se incrementó al $75,2 \%$ en el 2011.

En lo referente al empleo, la población económicamente activa (miles de personas) se incrementó poco de 13791,1 en el 2004 a
15949,1 en el 2011; la PEA ocupada ( miles de personas) sigue la misma tendencia ( Año 2004: 13059,8; Año 2011:15307,3). Sin embargo, en cuanto a la PEA ocupada según niveles de empleo, la categoría Adecuadamente empleada (porcentaje) se incrementó al doble $(44,8)$ en el 2011 respecto al $2004(22,2)$. Los avances en educación, son alentadores, ya que la tasa de analfabetismo de la población de 15 años a más ha disminuido, sin embargo, el porcentaje disminuyó de manera significativa en la zona urbana (Año 2005: 5,2; Año 2011: 4\% ) a diferencia de la rural ( Año 2005: 21,7 ; Año 2011: $17,4 \%$ ). El incremento del nivel educativo de la población de 15 a más años, sin nivel inicial ha disminuido en 3,8 puntos porcentuales en el 2011 con relación al 2005; en relación a la población de 15 a más años con primaria, secundaria y superior, esta última presenta un incremento mayor que las demás. Sin embargo, el gasto en educación como porcentaje del PBI en el año 2004 fue de 2,8 y en el 2011 fue de 2,9, con una variación mínima.

Los indicadores de salud en el Perú, también mejoraron, ya que la proporción de menores de 5 años con desnutrición crónica, experimentó una detracción, ya que en el 2006 el porcentaje era de 29,5, mientras que en el 2011 disminuyó a 19,5. Asimismo, el porcentaje de adolescentes de 15 a 19 años, que son madres o que están embarazadas por primera vez, no ha disminuido sino se ha mantenido (Año 2006: 12,2; Año 2011: 12,5).

\section{DISCUSIÓN}

Han transcurrido aproximadamente más de doce años y pareciera que la mayoría de los gobiernos regionales, efectivamente se han empoderado del rol que le compete, para lo cual han requerido de modificar sus diseños organizativos en el marco de la normativa vigente, empero, aún persiste en muchos casos una limitación de su autonomía administrativa, ya que la facultad de organizarse internamente, determinar y reglamentar los servicios públicos de su responsabilidad, siguen vulnerados y encasillados a una camisa de fuerza organizativa establecida por la mencionada ley. Por tanto, consideramos que para que un Gobierno Regional, pueda cumplir a cabalidad con su misión de promover el desarrollo de su región y brindar servicios claves para el bienestar de la población, se requiere no solo de optimizar sus procesos en su rol de gobierno, sino también optimizar la gerencia pública, con énfasis en la eficiente administración y gestión de los recursos públicos para asegurar el bienestar de la población, dejando de lado el gasto innecesario e irrelevante. A nuestro criterio, consideramos relevante definir dos roles macro en los gobiernos regionales, con igual relevancia y atención: gerenciar y gobernar. Al respecto, se observa muchas veces una inadecuada gerencia, lo que sin duda, menoscaba la gobernanza, situación que no se meritúa en la Secretaría de Gestión Pública de la Presidencia del Consejo de Ministros, ni en los propios gobiernos regionales, a fin de realizar un análisis estratégico de identificar fallas en la estructura o diseño organizacional definida en la Ley Orgánica de Gobiernos Regionales, o políticas centradas básicamente en mejorar los planes de gobierno y mejoramiento de prestación de servicios, pero soslayando la oportuna, eficiente y eficaz ejecución y asignación de recursos 
articulado con las carteras ministeriales, para lograr objetivos realmente de desarrollo regional, contrario sensu, estaríamos manteniendo el status quo. Conjuntamente, no menos importante, es la preocupación legítima de los gobernantes regionales como de la Secretaría de Gestión Pública de la Presidencia del Consejo de Ministros, de alcanzar los objetivos establecidos en la normativa vigente, como la mejora de los procesos y sistemas de administración para garantizar un buen servicio público, igualmente, la optimización de los trámites en las instituciones públicas en términos de simplificación administrativa y además una asignación puntual de competencias para precaver que las funciones se dupliquen y prevenir la omisión de las responsabilidades que cada funcionario debe asumir en el ejercicio de sus funciones.

El presupuesto público en el estado, es el medio por el cual se harán realidad los objetivos y necesidades que requiere la población, además no olvidemos que en el estado las necesidades son muchas y los recursos son escasos por eso quien desarrolle la administración financiera en una entidad pública, siempre ayudará sustancialmente a que la entidad se fortalezca y busque o cree nuevas formas de gestionar los recursos cuando advierta que una vez priorizado los mismos resultan insuficientes; y es ahí donde agregará valor a la gestión y se convertirá en una pieza clave en el entorno de la entidad y logrará cumplir con su rol de gerencia regional, lo que lamentablemente no ocurre en el Perú porque el diseño actual no lo ha previsto ni lo permite, estando inclusive prohibida por ley por eso que quien desee desarrollar la administración financiera de los recursos desde la Oficina de Administración incurriría en el delito de usurpación de funciones.

Tenemos el criterio tradicional que el área de presupuesto necesariamente debe estar a cargo de la gerencia de planeamiento, y que moverlo de ahí, para asignarse a otra gerencia sería no solo perder poder en el entorno institucional, sino que contra decidiría con la doctrina y postulados de la ciencia administrativa.

Existe una deficiente formulación y gestión de proyectos regionales que se debe principalmente a la inadecuada formulación y supervisión de los estudios de pre inversión y expedientes técnicos. Deficiente sostenibilidad de las obras debido a la limitada ejecución y supervisión de las mismas. No se asegura el presupuesto para la operación y mantenimiento de las obras terminadas. Existe una atomización de las inversiones. Se formulan pequeños estudios y no se prioriza la formulación de pocos proyectos integrales. Los procesos regulados por el sistema son complejos, engorrosos y en muchos casos, de difícil cumplimiento debido a que existe una regulación excesiva y rígida. Existe rotación excesiva en el personal de abastecimiento, lo que genera retrasos en la gestión. Los procesos de adquisición de bienes y servicios son obstaculizados por las demoras, impedimentos y formalidades del sistema.

En algunos casos el presupuesto no se puede ejecutar debido a que no hay suficiente presupuesto para acciones. Existe una gran desarticulación entre el Plan de Desarrollo Concertado (PDC), el Plan Estratégico Institucional (PEI), el Plan Operativo (POI), el Programa Multianual de Inversiones y el Presupuesto. La ejecución presupuestal se ve limitada por las inadecuadas normas sobre el calendario de compromisos, las cuales dificultan innecesariamente los desembolsos en la etapa de ejecución. Estas determinan que el calendario presupuestal debe cerrarse en la primera quincena del mes, limitando así la ejecución presupuestal a partir de la segunda quincena de cada mes. Hay una coexistencia de regímenes laborales diferentes.

La percepción del rol gerencial desplegado en los gobiernos regionales en cuanto a la contribución de prestación del servicio público es predominantemente de que constituye un rol desfasado que no contribuye a la gobernanza $(53,3 \%)$ y la relevancia que se le otorga al rol gerencial dentro del diseño organizacional por parte de los funcionarios públicos de prioritaria como parte del diseño organizacional $(46,7 \%)$.

Las razones que no permitirían una adecuada ejecución de los recursos en el ámbito de los Gobiernos Regionales según los funcionarios regionales, son mayormente porque prevalecen intereses políticos $(33,3 \%)$ y personal desmotivado (26,7\%). Los funcionarios públicos de los Gobiernos Regionales, en su mayoría consideran de muy necesaria $(63,3 \%)$ la vinculación entre la Secretaría de Descentralización del Presidencia del Consejo de Ministros y la Asamblea Nacional de Gobiernos Regionales para el cumplimiento del 
Rol de Gerencia en un Gobierno Regional.; y perciben que la razón principal por la que no se concreta dicha vinculación es porque mayormente depende de la decisión política del gobierno $(63,2 \%)$. La tendencia de transferencias gubernamentales a los gobiernos regionales 2007-2011, es incremental. EI porcentaje de ejecución presupuestal 2013-2014 que presentaron los gobiernos regionales en el Perú, oscila entre $83,50 \%$ (Madre de Dios) y $98,10 \%$ (Lima), no alcanzando en ningún caso el $100 \%$. La capacidad de ejecución global de la inversión pública en el Perú periodo agosto 2001 a febrero 2016, fue de franco incremento. El comportamiento de los indicadores de salud, acceso a telecomunicaciones, educación, empleo, y competitividad en el periodo 20062014 , es positivo en todos los casos. La inversión pública en términos de ejecutado /PIM en el Perú fue eficiente, en los tres niveles de gobierno (nacional, regional y local) ya que se incrementó considerablemente en el 2014 respecto al 2007. Con el presente trabajo de investigación se formula un modelo de gerencia para optimizar la gestión y administración de recursos en los gobiernos regionales, con un nuevo enfoque organizacional.

Presentado: Marzo 2017

Aceptado: Junio 2017

\section{REFERENCIAS BIBLIOGRÁFICAS}

Angeles C ( 2012) La descentralización en el Perú: Un proceso inconcluso, Facultad de Derecho y Ciencia Política de la Universidad Peruana Los Andes, Lima.

Banco Mundial (2014) Datos de libre acceso del Banco $\begin{array}{llllll}M & u & n & d & i & a\end{array}$ url:http://datos.bancomundial.org/[Consulta 23.07.15].

Castilla L. (2012) Perspectivas para el desarrollo:Finanzas Públicas y Desarrollo en A m é $r$ i c a $L$ a $t$ i $n$ a $U R L$ : https://www.up.edu.pe/carrera/economia/Site A $\mathrm{s}$ s e $\mathrm{t} \quad \mathrm{s} / \mathrm{L}$ i $\mathrm{s}$ t $\mathrm{s} / \mathrm{N}$ OT_Noticia/NewForm/Presentaci\%C3\%B3n $\begin{array}{llllllllllllll}\% & 2 & 0 & M & \text { i } & n & \text { i } & s & t & r & 0 & \% & 2\end{array}$ 0de $\% 20$ Econom\%C3\%ADa.pdf

Guzmán J. y Tortolero F. (2013) La gerencia pública en la construcción de una nueva relación entre ciudadanos y las instituciones $\mathrm{gub}$ e r n a m e $\mathrm{t}$ ale s e $\mathrm{n}$ Venezuela.URL:http://ri.bib.udo.edu.ve/bitstre a m/123456789/3619/1/Tes is GuzmanJyTortoleroF.pdf

Jiménez H. (2010) La gestión de intereses en la administración pública peruana .Tesis para optar el grado de maestro en gerencia pública [En línea] Disponible en http://cybertesis.uni.edu.pe/bitstream/uni/213/ 1/jimenez_sh.

Levitsky, Steven: "Una paradoja peruana". En: http://www.larepublica.pe/columnistas/aproxi maciones/una-paradoja-peruana-12-05-2012. 13 de mayo 2012.

Ley $N^{\circ} 27680$ (2002) Ley de Reforma Constitucional del Capítulo XIV del Título IV sobre Descentralización, Lima .

Llona M. (2013) La modernización de la gestión pública: los retos actuales http://escuela.pucp.edu.pe/gobierno/images/d ocumentos/seminario/2011/modernizacin $\% 20$ del\%20estado\%20y\%20ges tin\%20pblica.pdf [ Consulta 4.078.15].

Morales O., Barrera A., Rodríguez M. , Romero C. y
Távara R. ( 2014) Modelo de gestión de la innovación para los gobiernos locales del Perú [ En Iínea ] Disponible en http://www.esan.edu.pe/publicaciones/2014/0 $2 / 27 / \mathrm{s}$ e $\mathrm{r}$ i e $-g$ e $r$ encia_desarrollo_35_modelo_gestion_innova cion_gobierno s_locales_peru.pdf [Consulta 23.07.15].

Oyarce H. (2012) Gerencia Pública http://www.cepal.org/ilpes/noticias/paginas/4/ 45114/Gerenci a_Publica2011_HOYARCE.pdf

Presidencia del Consejo de Ministros. Secretaría de Descentralización (2012) Plan Nacional de Descentralización 2012-2016, Lima.

Peraza A. (2010) Propuesta de un modelo gerencial estratégico socialmente responsable basado en el gobierno electrónico para la gestión de los gobiernos locales en el estado Aragua

Perú - Presidencia del Consejo de Ministros (2013) Política Nacional de Modernización de la G e s t i ón $\mathrm{P}$ ú b I i c a a I 20021 http://inst.servir.gob.pe/index.php/es/noticiasy-eventos-/1587-modernizacion-de-lagestion-publica-en-el- peru.html[Consulta 15.07.15].

Presidencia del Consejo de Ministros. Secretaría de Descentralización (2012) Plan Nacional de Descentralización 2012-2016, Lima.

Romero M. (2010) Reforma de Estado, Gerencia Pública Social y Organizaciones No Lucrativas. Reflexiones en torno a Venezuela. http://tesis.luz.edu.ve/tde_busca/arquivo.php ?codArquivo $=2$

USAID DEL PUEBLO DE LOS ESTADOS UNIDOS DE AMÉRICA(2011) Introducción a la buena gestión pública y la gestión por resultados. Un aspecto clave para las gestiones de las

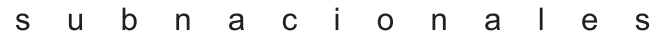
http://www.regionsanmartin.gob.pe/administra

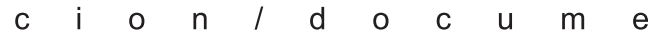
ntos_transparencia/doc_transparencia261.pdf 